\title{
ACCELERATED NONCARTESIAN SENSE RECONSTRUCTION USING A MAJORIZE-MINIMIZE ALGORITHM COMBINING VARIABLE-SPLITTING
}

\author{
Sathish Ramani and Jeffrey A. Fessler \\ EECS Department, University of Michigan, Ann Arbor, MI 48109-2122, USA.
}

\begin{abstract}
Magnetic resonance imaging (MRI) provides great flexibility in the choice of k-space sampling trajectories. NonCartesian trajectories exhibit several advantages over Cartesian ones but are less amenable to FFT-based manipulation of k-space data. Thus, existing iterative reconstruction methods for nonCartesian trajectories require relatively more computation (interpolation/gridding in addition to FFTs) and can be slow, especially for (undersampled) parallel MRI. In this work, we focus on SENSE-based regularized image reconstruction for nonCartesian trajectories and propose a majorize-minimize approach where we first majorize the SENSE data-fidelity term with a quadratic form involving a symmetric positive definite circulant matrix. For the minimization step, we apply a suitable variable splitting (VS) strategy combined with the augmented Lagrangian framework and alternating minimization that together decouple the circulant matrix from coil sensitivities and the regularizer. The resulting iterative algorithm admits simple update steps, is amenable to FFT-based matrix inversions due in part to the circulant matrix in the majorizer and provides a natural framework for incorporating a two-step procedure for acceleration. Simulations indicate that the proposed algorithm converges faster than some state-of-the-art VSbased iterative image reconstruction methods for the same problem.
\end{abstract}

Index Terms - SENSitivity Encoding (SENSE), NonCartesian trajectories, Majorize-minimize, Variable-splitting, Augmented Lagrangian.

\section{INTRODUCTION}

Magnetic resonance imaging (MRI) is a versatile imaging technique that provides great flexibility in the choice of sampling trajectories for acquiring measurements in $\mathrm{k}$-space. NonCartesian trajectories possess several advantages over Cartesian ones such as efficient coverage of $\mathrm{k}$-space for a given number of phase encodes and robustness to motion and off-resonance effects [1]. However, for nonCartesian trajectories, image reconstruction can no longer be accomplished with DFT / FFT alone and requires additional operations (e.g., nonuniform FFT (NUFFT) [2] performs interpolation/gridding in addition to FFTs) that can add to compute time during (iterative) reconstruction.

In this work, we focus on SENSitivity Encoding (SENSE) based regularized image reconstruction-Regularized SENSEsince SENSE can tackle arbitrary nonCartesian trajectories. We minimize a cost function $J$ composed of a SENSE-type data-fidelity term [3] and a convex regularizer $[4,5]$. To circumvent repetitive use of NUFFT-type operations when iteratively minimizing $J$, we propose to use a majorize-minimize (MM) approach where we first design a majorizer $J_{\text {maj }}$ for $J$ that involves a symmetric positive definite circulant matrix. Next, we minimize $J_{\text {maj }}$ using an appropriate variable splitting (VS) strategy combined with the augmented Lagrangian (AL) framework and alternating minimization (AM) similar to that in [5]. This leads to an iterative MAMAL

This work was supported by the National Institutes of Health under Grant P01 CA87634.
(MAjorize-Minimize AL) algorithm that admits simple update steps and FFT-based matrix inversions due to the circulant matrix in $J_{\text {maj }}$. We further accelerate MAMAL using a two-step update (TS) strategy similar to that discussed in [6, Sec. 4] leading to MALTS (Majorizeminimize AL TS). Simulation results indicate that MALTS converges to a minimizer of $J$ faster than state-of-the-art VS-based iterative reconstruction techniques such as the split-Bregman (SB) [7] and the Bregman operator splitting (BOS) [8] methods that are applicable to Regularized SENSE.

\section{REGULARIZED SENSE}

SENSE provides a simple framework for incorporating regularization that is necessary to reduce aliasing artifacts and noise at high reduction (undersampling) factors [4]. Regularized SENSE reconstruction can be formulated as an optimization problem $[4,5]$,

$$
\begin{aligned}
& \mathbf{P 0}: \mathbf{x}^{(\star)} \triangleq \arg \min _{\mathbf{x}}\{J(\mathbf{x}) \triangleq D(\mathbf{d}, \mathbf{S} \mathbf{x})+\lambda \Psi(\mathbf{R} \mathbf{x})\}, \\
& D(\mathbf{d}, \mathbf{S} \mathbf{x}) \triangleq\|\mathbf{d}-\mathbf{F S} \mathbf{S}\|_{2}^{2},
\end{aligned}
$$

where $\mathbf{x}^{(\star)} \in \mathbb{C}^{N}$ is the reconstructed image, $\mathbf{S} \triangleq\left[\mathbf{S}_{1}^{\prime} \cdots \mathbf{S}_{L}^{\prime}\right]^{\prime}$, $\mathbf{S}_{l} \in \mathbb{C}^{N \times N}$ is a diagonal matrix of sensitivity map of the $l$ th coil, $l=1, \ldots, L, \mathbf{F}=\mathbf{I}_{L} \otimes \mathbf{F}_{\mathrm{u}}, \mathbf{F}_{\mathrm{u}} \in \mathbb{C}^{M \times N}$ is the Fourier encoding matrix corresponding to a nonCartesian (undersampled) $\mathrm{k}$ space trajectory, $\otimes$ denotes the Kronecker product, $\mathbf{d} \in \mathbb{C}^{M L}$ is the acquired (undersampled) measurements from all $L$ coils, $(\cdot)^{\prime}$ represents (hermitian) transpose of a (complex) vector or matrix, $\mathbf{I}_{L}$ is the identity matrix of size $L, \Psi$ is a suitable convex regularizer, $\mathbf{R} \in \mathbb{R}^{P \times N}, P \geq N$, represents a regularization operator (e.g., finite differences) and $\lambda>0$ is the regularization parameter. In (1), we ignore field inhomogeneity and relaxation effects and assume that a suitable whitening procedure has been applied for tackling correlated noise [3, App. B]. Problem P0 is nontrivial and demands computation-intensive nonlinear optimization when $\Psi$ is a nonquadratic regularizer, e.g., edge-preserving total variation (TV) or an $\ell_{1}$-regularizer that promotes sparsity. VS based iterative algorithms [5,7] have recently gained popularity for (parallel) MRI reconstruction as they can effectively handle nonquadratic $\Psi$ including $\mathrm{TV}$ and $\ell_{1}$-regularizers.

\subsection{The Split-Bregman Algorithm [7]}

The SB method is a VS-based scheme that separates $\Psi$ from $D$ (1) using an auxiliary constraint variable $\mathbf{v}$ and converts $\mathbf{P 0}$ into an equivalent constrained problem of the form $\min _{\mathbf{x}, \mathbf{v}}\{D(\mathbf{d}, \mathbf{S} \mathbf{x})+$ $\lambda \Psi(\mathbf{v})\}$ s.t. $\mathbf{v}=\mathbf{R x}$. This problem is then solved using Bregman iterations along with an AM scheme [7]. The resulting SB algorithm requires solving a linear system involving $\mathbf{G}_{\mu} \triangleq \mathbf{S}^{\prime} \mathbf{F}^{\prime} \mathbf{F} \mathbf{S}+\mu \mathbf{R}^{\top} \mathbf{R}$ for some $\mu>0$ [5, Sec. IV-B] at each iteration. Although the conjugate gradient $(\mathrm{CG})$ method can be used, it may converge slowly depending on the condition of $\mathbf{G}_{\mu}$. This problem is further compounded by the shift-variant nature of $\mathbf{S}^{\prime} \mathbf{F}^{\prime} \mathbf{F S}$ (for both Cartesian and nonCartesian trajectories) that is not easily amenable to efficient preconditioning. 


\subsection{An Augmented Lagrangian Algorithm [5]}

To get around $\mathbf{G}_{\mu}$ in SB, we recently proposed a VS scheme in [5, Sec. IV-B] that converts $\mathbf{P 0}$ to $\min _{\mathbf{x}, \mathbf{u}, \mathbf{v}, \mathbf{w}}\{D(\mathbf{d}, \mathbf{u})+\lambda \Psi(\mathbf{v})\}$ s.t. $\mathbf{u}=\mathbf{S} \mathbf{x}, \mathbf{v}=\mathbf{R} \mathbf{w}, \mathbf{w}=\mathbf{x}$. When solved using the AL framework and AM, this constrained problem led to an iterative algorithm involving the inversion of $\mathbf{H}_{\mu} \triangleq \mathbf{I}_{L} \otimes\left(\mathbf{A}_{\mu} \triangleq \mathbf{F}_{\mathrm{u}}^{\prime} \mathbf{F}_{\mathrm{u}}+\mu \mathbf{I}_{M}\right)$ (among other simple update steps comparable to those in the SB algorithm) at each iteration. The advantage here is that $\mathbf{A}_{\mu}$ is exactly invertible for Cartesian trajectories using FFTs; this feature enabled accelerated convergence of this algorithm over SB [5]. For nonCartesian trajectories however there is no direct (one-step) method for inverting $\mathbf{A}_{\mu}$; CG can be used with a suitable circulant preconditioner since $\mathbf{A}_{\mu}$ is Toeplitz $\left(\mathbf{F}_{\mathrm{u}}^{\prime} \mathbf{F}_{\mathrm{u}}\right.$ is Toeplitz for nonCartesian trajectories ignoring field inhomogeneity and relaxation effects). However, one has to apply such a CG-solver $L$ times (due to the $\mathbf{I}_{L}$ in $\mathbf{H}_{\mu}$ ), which can be time consuming.

\section{PROPOSED APPROACH}

Our aim in this work is to design an (iterative) algorithm for solving P0 (1) that admits exact inner update steps for nonCartesian trajectories. To this end, we use a majorize-minimize approach and construct a majorizer $J_{\text {maj }}$ such that

$$
J(\mathbf{x}) \leq J_{\text {maj }}\left(\mathbf{x}, \mathbf{x}^{(j)}\right), \forall \mathbf{x} \neq \mathbf{x}^{(j)},
$$

with equality at $\mathbf{x}=\mathbf{x}^{(j)}$. So the iterative scheme

$$
\mathbf{x}^{(j+1)}=\arg \min _{\mathbf{x}} J_{\operatorname{maj}}\left(\mathbf{x}, \mathbf{x}^{(j)}\right)
$$

is guaranteed to (monotonically) decrease $J$ in (1). In practice, (4) need not be carried out explicitly; a decrease in $J_{\text {maj }}$ is sufficient to ensure a decrease of $J$. Below, we describe a suitable $J_{\text {maj }}$ and describe a minimization scheme for (4) that is based on a VS strategy combined with the AL framework and AM similar to that in [5]. Throughout the paper, we follow the convention in [9]: if $f: \mathbb{C}^{N} \rightarrow$ $\Omega(\Omega \equiv \mathbb{R}$ or $\mathbb{C})$ is individually analytic with respect to $\mathbf{x} \in \mathbb{C}^{N}$ and $\mathbf{x}^{*}$ (its complex conjugate), gradient of $f$ with respect to $\mathbf{x}, \nabla_{\mathbf{x}} f$, can be evaluated treating $\mathbf{x}^{*}$ as a constant and vice-versa [9].

\subsection{Majorization of $J$}

We begin by considering $\mathbf{C} \triangleq \mathbf{I}_{L} \otimes \mathbf{C}_{\mathrm{u}}$, where $\mathbf{C}_{\mathrm{u}} \in \mathbb{C}^{N \times N}$ is a circulant matrix such that $\mathbf{C}_{\mathrm{u}}^{\prime} \mathbf{C}_{\mathrm{u}} \succeq \mathbf{F}_{\mathrm{u}}^{\prime} \mathbf{F}_{\mathrm{u}}$. We discuss the choice of $\mathbf{C}_{\mathrm{u}}$ in Sec. 3.4. It is easy to see that $\mathbf{S}^{\prime} \mathbf{C}^{\prime} \mathbf{C}^{\prime} \mathbf{S} \succeq \mathbf{S}^{\prime} \mathbf{F}^{\prime} \mathbf{F}^{\prime} \mathbf{S}$ and

$$
\begin{aligned}
D(\mathbf{d}, \mathbf{S x}) \leq & D\left(\mathbf{d}, \mathbf{S} \mathbf{x}^{(j)}\right)+2 \mathcal{R}\left\{\left.\left(\mathbf{x}-\mathbf{x}^{(j)}\right)^{\prime} \nabla_{\mathbf{t}^{*}} D(\mathbf{d}, \mathbf{S t})\right|_{\mathbf{t}=\mathbf{x}}\right\} \\
& +\left(\mathbf{x}-\mathbf{x}^{(j)}\right)^{\prime} \mathbf{S}^{\prime} \mathbf{C}^{\prime} \mathbf{C S}\left(\mathbf{x}-\mathbf{x}^{(j)}\right)
\end{aligned}
$$

where equality holds in (5) at $\mathbf{x}=\mathbf{x}^{(j)}$. The rhs of (5) can be simplified to obtain

$$
\begin{aligned}
D_{\text {maj }}\left(\mathbf{d}, \mathbf{S x}, \mathbf{x}^{(j)}\right) \triangleq & 2 \mathcal{R}\left\{-\mathbf{x}^{\prime} \mathbf{S}^{\prime}\left[\mathbf{F}^{\prime} \mathbf{d}+\left(\mathbf{C}^{\prime} \mathbf{C}-\mathbf{F}^{\prime} \mathbf{F}\right) \mathbf{S} \mathbf{x}^{(j)}\right]\right\} \\
& +\mathbf{x}^{\prime} \mathbf{S}^{\prime} \mathbf{C}^{\prime} \mathbf{C S} \mathbf{x}+C\left(\mathbf{x}^{(j)}\right)
\end{aligned}
$$

where $C\left(\mathbf{x}^{(j)}\right)$ is a constant irrelevant for optimization and will be ignored henceforth. Thus, setting

$$
J_{\text {maj }}\left(\mathbf{x}, \mathbf{x}^{(j)}\right) \triangleq D_{\text {maj }}\left(\mathbf{d}, \mathbf{S x}, \mathbf{x}^{(j)}\right)+\lambda \Psi(\mathbf{R} \mathbf{x}),
$$

we obtain a majorizer $J_{\text {maj }}$ that satisfies (3). Next, we attack the minimization in (4) for $J_{\text {maj }}$ in (7).

\subsection{Minimization of $J_{\mathrm{maj}}$}

The minimization in (4) for $J_{\text {maj }}$ in (7) is still a nontrivial task, so we apply the VS strategy in [5, Sec. IV-B] and convert (4) in to the following equivalent constrained problem

$$
\begin{gathered}
\min _{\mathbf{z}}\left\{D_{\text {maj }}\left(\mathbf{d}, \mathbf{u}, \mathbf{x}^{(j)}\right)+\lambda \Psi(\mathbf{v})\right\} \text { s.t. } \mathbf{B z}=\mathbf{0}, \\
\mathbf{B} \triangleq\left[\begin{array}{cccc}
\mathbf{I}_{N L} & \mathbf{0} & \mathbf{0} & -\mathbf{S} \\
\mathbf{0} & \mathbf{I}_{P} & -\mathbf{R} & \mathbf{0} \\
\mathbf{0} & \mathbf{0} & \mathbf{I}_{N} & -\mathbf{I}_{N}
\end{array}\right], \mathbf{z} \triangleq\left[\begin{array}{c}
\mathbf{u} \\
\mathbf{v} \\
\mathbf{w} \\
\mathbf{x}
\end{array}\right] .
\end{gathered}
$$

Then we use the AL framework with AM as elucidated in [5, Sec. IVB] to solve (8). Specifically, we construct the AL function as

$$
\mathcal{L}(\mathbf{z}, \boldsymbol{\eta}) \triangleq D_{\text {maj }}\left(\mathbf{d}, \mathbf{u}, \mathbf{x}^{(j)}\right)+\lambda \Psi(\mathbf{v})+\mu\|\mathbf{B} \mathbf{z}-\boldsymbol{\eta}\|_{\boldsymbol{\Lambda}}^{2},
$$

where $\boldsymbol{\eta} \triangleq\left[\boldsymbol{\rho}^{\prime} \boldsymbol{\vartheta}^{\prime} \boldsymbol{\omega}^{\prime}\right]^{\prime}$ is the Lagrange-multiplier-like vector for the constraints in (8), $\boldsymbol{\Lambda} \triangleq \operatorname{diag}\left\{\mathbf{I}_{N L}, \nu \mathbf{I}_{P}, \tau \mathbf{I}_{N}\right\}$ and $\mu, \nu, \tau>0$ are $\mathrm{AL}$ penalty parameters. The AL technique with AM in [5, Sec. IVB] leads to the following iterative scheme for solving (8): at $j$ th iteration of (4), we set $\mathbf{x}^{(j, 1)} \leftarrow \mathbf{x}^{(j)}$ and run the following for $k=$ $1, \ldots, K$ :

$$
\begin{aligned}
\mathbf{u}^{(j, k+1)} & =\left(\mathbf{C}^{\prime} \mathbf{C}+\mu \mathbf{I}_{N L}\right)^{-1}\left[\begin{array}{l}
\mathbf{F}^{\prime} \mathbf{d}+\left(\mathbf{C}^{\prime} \mathbf{C}-\mathbf{F}^{\prime} \mathbf{F}\right) \mathbf{S} \mathbf{x}^{(j)} \\
+\mu\left(\mathbf{S} \mathbf{x}^{(j, k)}+\boldsymbol{\rho}^{(j, k)}\right)
\end{array}\right], \\
\mathbf{v}^{(j, k+1)} & =\arg \min _{\mathbf{v}} \frac{\lambda}{\mu \nu} \Psi(\mathbf{v})+\left\|\mathbf{v}-\mathbf{R} \mathbf{w}^{(j, k)}-\boldsymbol{\vartheta}^{(j, k)}\right\|_{2}^{2}, \\
\mathbf{w}^{(j, k+1)} & =\left(\mathbf{R}^{\prime} \mathbf{R}+\tau / \nu \mathbf{I}_{N}\right)^{-1}\left[\begin{array}{l}
\mathbf{R}^{\prime}\left(\mathbf{v}^{(j, k+1)}-\boldsymbol{\vartheta}^{(j, k)}\right) \\
+\tau / \nu\left(\mathbf{x}^{(j, k)}+\boldsymbol{\omega}^{(j, k)}\right)
\end{array}\right], \\
\mathbf{x}^{(j, k+1)} & =\left(\mathbf{S}^{\prime} \mathbf{S}+\tau \mathbf{I}_{N}\right)^{-1}\left[\begin{array}{c}
\mathbf{S}^{\prime}\left(\mathbf{u}^{(j, k+1)}-\boldsymbol{\rho}^{(j, k)}\right) \\
+\tau\left(\mathbf{w}^{(j, k+1)}-\boldsymbol{\omega}^{(j, k)}\right)
\end{array}\right], \\
\boldsymbol{\eta}^{(j, k+1)} & =\boldsymbol{\eta}^{(j, k)}-\mathbf{B} \mathbf{z}^{(j, k+1)}
\end{aligned}
$$

The $\mathbf{u}^{(k+1)}$ update (11) can be implemented using FFTs since $\mathbf{C}^{\prime} \mathbf{C}$ is circulant. Similarly, if $\mathbf{R}^{\prime} \mathbf{R}$ is circulant (finite differences or frames with periodic boundary extension), $\mathbf{w}^{(k+1)}$ (13) can also be computed using FFTs. The $\mathbf{x}^{(j, k+1)}$ update (14) is trivial since $\mathbf{S}^{\prime} \mathbf{S}$ is diagonal and finally, the $\mathbf{v}^{(k+1)}$ update (12) admits a closed-form solution for a variety of regularizers $\Psi$ [5, Sec. IV-A], e.g., scalarshrinkage for $\ell_{1}$-regularization and vector-shrinkage for TV $[5,7]$.

Equations (4) and (11)-(15) constitute our MAMAL algorithm for solving P0 (1). The main steps (11)-(14) can be implemented either directly (closed-form) or efficiently unlike the SB or AL algorithms discussed in Secs. 2.1 and 2.2, respectively. At the end of $K$ iterations of (11)-(15), we set $\mathbf{x}^{(j+1)} \leftarrow \mathbf{x}^{(j, K)}$. We also found in our experiments that it is better to set $\boldsymbol{\eta}^{(j+1,1)} \leftarrow \boldsymbol{\eta}^{(j, K)}$ (warm-starting) rather than $\boldsymbol{\eta}^{(j+1,1)}=\mathbf{0}$ before each $j$-iteration (4).

\subsection{Acceleration Using a Two-Step Procedure}

The MM feature of MAMAL provides a natural framework for incorporating the two-step (TS) procedure discussed in [6, Sec. 4]. To combine MAMAL with TS we replace (4) with the following:

$$
\begin{aligned}
\mathbf{x}^{(j+1)} & =\arg \min _{\mathbf{x}} J_{\text {maj }}\left(\mathbf{x}, \mathbf{y}^{(j)}\right), \\
\mathbf{y}^{(j)} & =\mathbf{x}^{(j)}+\left(a_{j}-1\right) / a_{j+1}\left(\mathbf{x}^{(j)}-\mathbf{x}^{(j-1)}\right),
\end{aligned}
$$

where $a_{j+1}=\left[1+\sqrt{1+4 a_{j}^{2}}\right] / 2$ and $a_{1}=1$. The above TS scheme evaluates $J_{\text {maj }}$ at $\mathbf{y}^{(j)}$ that is a combination of two iterates, $\mathbf{x}^{(j)}$ and $\mathbf{x}^{(j-1)}$, and hence the phrase "two-step." Using the above TS procedure simply amounts to replacing $\mathbf{x}^{(j)}$ in the rhs of (11) with $\mathbf{y}^{(j)}$ from (17). We demonstrate in our experiments that combining MAMAL with TS (MALTS) accelerates its convergence. 

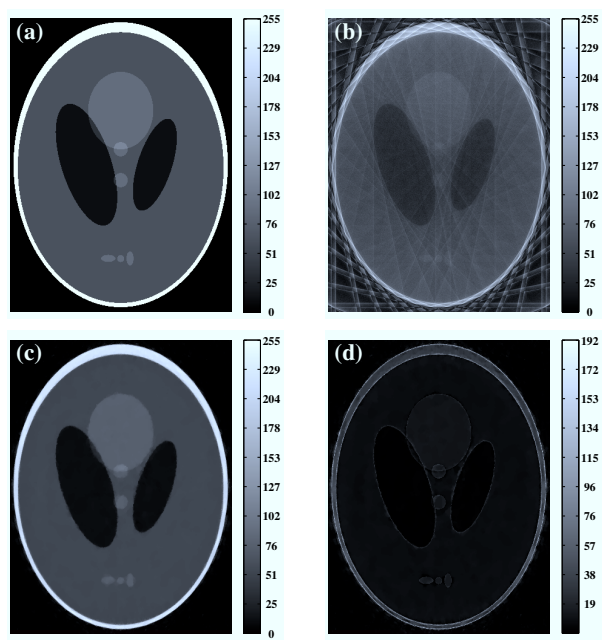

Fig. 1. Simulation with the Analytical Shepp-Logan phantom [10]: (a) Discretized $(512 \times 512)$ noisefree phantom; (b) SoS of conjugate phase reconstruction with density compensation (initial estimate $\mathbf{x}^{(1)}$ ); (c) TV-regularized SENSE reconstruction result [solution $\mathbf{x}^{(\star)}$ of P0 (1)]; (d) Absolute difference between (a) and (c).

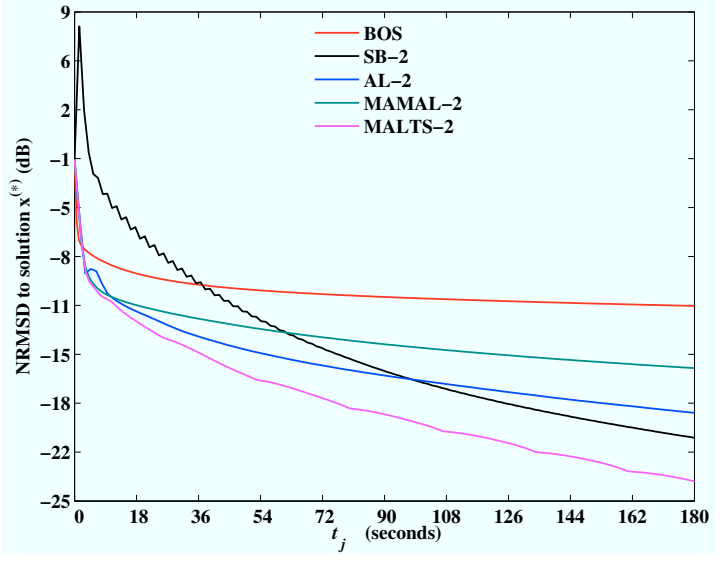

Fig. 2. Plot of NRMSD between $\mathbf{x}^{(j)}$ and a solution $\mathbf{x}^{(\star)}$ of P0 (1) for simulation with the Analytical Shepp-Logan phantom [10].

\subsection{Choice of $\mathbf{C}_{u}$}

Ideally, we would to like use $\mathbf{C}_{\mathrm{u}}=\arg \min _{\mathbf{C}} \operatorname{tr}\left\{\mathbf{C}^{\prime} \mathbf{C}\right\}$ s.t. $\mathbf{C}^{\prime} \mathbf{C} \succeq$ $\mathbf{F}^{\prime} \mathbf{F}$, but this is difficult to obtain. So we use a simpler alternative where we start from $\mathbf{C}_{\text {Frob }} \succeq \mathbf{0}$, the optimal circulant approximation to $\mathbf{F}_{\mathrm{u}}^{\prime} \mathbf{F}_{\mathrm{u}}$ in Frobenius-norm [11], and construct $\mathbf{C}_{\kappa} \succ \mathbf{0}$ from $\mathbf{C}_{\text {Frob }}$ with condition number $\kappa$ (by increasing the smallest eigenvalue of $\mathbf{C}_{\text {Frob }}$ accordingly). We used a condition number of $\kappa=100$ in all experiments. We then set $\mathbf{C}_{\mathrm{u}} \triangleq\left(\alpha \mathbf{C}_{\kappa}\right)^{\frac{1}{2}}$, where $\alpha$ satisfies $\alpha \mathbf{C}_{\kappa} \succeq \mathbf{F}_{\mathrm{u}}^{\prime} \mathbf{F}_{\mathrm{u}}$. Such an $\alpha$ is only trajectory dependent (via $\mathbf{F}_{\mathrm{u}}$ ) and can be easily precomputed using the Power method.

\section{SIMULATIONS}

We implemented and compared the following algorithms in Matlab:

1. BOS: Bregman operator splitting method [8],

2. SB- $n$ [7] with $n$ PCG iterations involving $\left(\mathbf{C}_{\text {Frob }}+\mu \mathbf{R}^{\prime} \mathbf{R}\right)^{-1}$ as the preconditioner for solving the linear system mentioned in Sec. 2.1,

3. AL $-n\left[5\right.$, Sec. IV-B] with $n$ PCG iterations involving $\left(\mathbf{C}_{\text {Frob }}+\right.$ $\left.\mu \mathbf{I}_{N}\right)^{-1}$ as the preconditioner for solving the linear system mentioned in Sec. 2.2,

\section{Proposed MAMAL $-K$ with $K$ iterations of (11)-(15), \\ 5. Proposed MALTS $-K$ with $K$ iterations of (11)-(15).}

Since we run only a finite number of iterations, $K$, of (11)-(15), MALTS can be sensitive to the TS procedure, so we set $a_{j}=1$ (17) for $j=25 m, m \geq 1$, to avoid instabilities arising due to (17). However, MAMAL is guaranteed to decrease the cost $J$ in (1) monotonically even with a finite $K$ as discussed in Sec. 3 .

All of the above algorithms are based on VS and AL strategies, so they require selection of appropriate AL penalty parameters (that do not influence the solution of PO but govern only their convergence speeds), e.g., $\mu>0$ in $\mathrm{SB}$ and $\mathrm{BOS}$, and $\mu, \nu, \tau>0$ in AL, MAMAL and MALTS. To be fair to all algorithms in the selection of AL penalty parameters, we focussed on $\mathbf{v}^{(\cdot)}$ update (12) that is common to all of the above algorithms and set $\mu=\lambda / \gamma$ for SB and BOS and $\mu=\lambda /(\gamma \nu)$ for AL, MAMAL and MALTS for some $\gamma>0$ discussed below. These choices ensured that $\Psi$ in the $\mathbf{v}^{(\cdot)}$ update (12) was weighted by the same $\gamma$ for all algorithms. For AL, MAMAL and MALTS, we further fixed $\tau=\operatorname{median}\left\{s_{i}\right\}$, where $\left\{s_{i}\right\}$ are the diagonal elements of $\mathbf{S}^{\prime} \mathbf{S}$ and $\nu=\sigma_{\max }^{-1}\left\{\mathbf{R}^{\prime} \mathbf{R}\right\}$, where $\sigma_{\max }\left\{\mathbf{R}^{\prime} \mathbf{R}\right\}$ is the maximum eigenvalue of $\mathbf{R}^{\prime} \mathbf{R}$, in all our experiments. We ran the algorithms on a PC with quad-core $3.07 \mathrm{GHz}$ Intel Xeon processor. We quantified the convergence speed of the algorithms in terms of the normalized root mean squared distance (NRMSD) between an iterate $\mathbf{x}^{(j)}$ and a solution $\mathbf{x}^{(\star)}(1)$ computed as $20 \log _{10}\left(\left\|\mathbf{x}^{(j)}-\mathbf{x}^{(\star)}\right\|_{2} /\left\|\mathbf{x}^{(\star)}\right\|_{2}\right)$. We obtained $\mathbf{x}^{(\star)}$ numerically by running 1000 iterations of $S B-10$ in all experiments. In all algorithms, we used NUFFT [2] for products with $\mathbf{F}_{\mathrm{u}}$, while for those with $\mathbf{F}_{\mathrm{u}}^{\prime} \mathbf{F}_{\mathrm{u}}$, we used the "embedding-toeplitz-in-circulant" trick, i.e., $\mathbf{F}_{\mathrm{u}}^{\prime} \mathbf{F}_{\mathrm{u}}=\mathbf{Z}^{\prime} \mathbf{Q Z}$, where $\mathbf{Z}$ is a $2 N \times N$ zero-padding matrix and $\mathbf{Q}$ is an appropriate $2 N \times 2 N$ circulant matrix [12]. We used a sum-of-squares (SoS) combination of the conjugate phase reconstruction (CPR) $\mathbf{F}^{\prime} \mathbf{W} \mathbf{d}$ as the initialization $\mathbf{x}^{(1)}$ for all algorithms, where $\mathbf{W}$ is a diagonal matrix of suitable density compensation weights. We set $\mathbf{u}^{(1)}=\mathbf{S} \mathbf{x}^{(1)}, \mathbf{w}^{(1)}=\mathbf{x}^{(1)}, \mathbf{v}^{(1)}=\mathbf{R} \mathbf{w}^{(1)}$ and $\boldsymbol{\eta}^{(1)}=\mathbf{0}$ in (11)-(15).

We used a radial trajectory with 16 spokes each with 512 samples and generated noisy data $\mathbf{d}$ (1) of $40 \mathrm{~dB}$ SNR for $L=8$ coils using simulated coil-sensitivities [13] and the analytical Shepp-Logan phantom $(\mathrm{FOV}=15 \mathrm{~cm})$ [10]. We also simulated $32 \times 32$ Cartesian noisy k-space data $\mathbf{d}_{\text {cart }}$ of same SNR for all $L=8$ coils and used $\mathbf{d}_{\text {cart }}$ to estimate $\mathbf{S}$ (1) with the method described in [14]. We used the TV regularizer (with finite differences for $\mathbf{R}$ ) for which we set $\gamma$ discussed earlier such that $90 \%$ of the elements of $\left(\mathbf{R} \mathbf{w}^{(1)}+\boldsymbol{\vartheta}^{(1)}\right)$ were nonzero after vector-shrinkage operation at (12) for TV. The $512 \times 512$ TV-regularized solution $\mathbf{x}^{(\star)}$ to $\mathbf{P 0}(1)$, Fig. $1 \mathrm{c}$, has less artifacts than CPR, Fig. 1b, and closely resembles the oracle noisefree (discretized) phantom, Fig. 1a. The difference image, Fig. 1d, indicates that $\mathbf{x}^{(\star)}$ has captured most of the smooth details of the original phantom, but has some (structured) residual error. We ran the algorithms listed above, computed and plotted NRMSD as a function of run-time for each algorithm in Fig. 2. The proposed MAMAL is faster than both $\mathrm{SB}$ and BOS and is comparable to AL during the initial few iterations. MALTS decreases NRMSD relatively rapidly compared to all algorithms providing accelerated convergence in this experiment.

In the next experiment, we generated noisy data of $50 \mathrm{~dB}$ SNR for $L=8$ coils (using simulated coil-sensitivities [13]) by applying NUFFT to a $2048 \times 2048 T_{2}$-weighted image (interpolated from a noisefree $256 \times 256$ Brainweb [15] $T_{2}$-weighted MR image, $\mathrm{FOV}=25 \mathrm{~cm}$ ). We used a variable-density spiral with 5 interleaves (reduction factor $=5 / 3$ at the center and 5 at outer $\mathrm{k}$-space). We again simulated $32 \times 32$ Cartesian noisy k-space data $\mathbf{d}_{\text {cart }}$ of same SNR for all $L=8$ coils and used the method described in [14] 

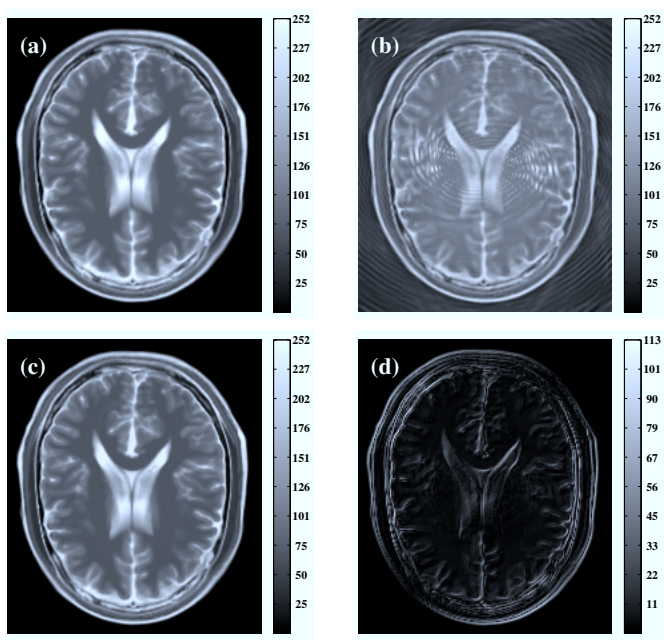

Fig. 3. Simulation with a BrainWeb [15] $T_{2}$-weighted MRI image: (a) Noisefree $(256 \times 256) T_{2}$-weighted image; (b) SoS of conjugate phase reconstruction with density compensation (initial estimate $\mathbf{x}^{(1)}$ ); (c) $\ell_{1}$-regularized SENSE reconstruction result [solution $\mathbf{x}^{(\star)}$ of P0 (1)]; (d) Absolute difference between (a) and (c).

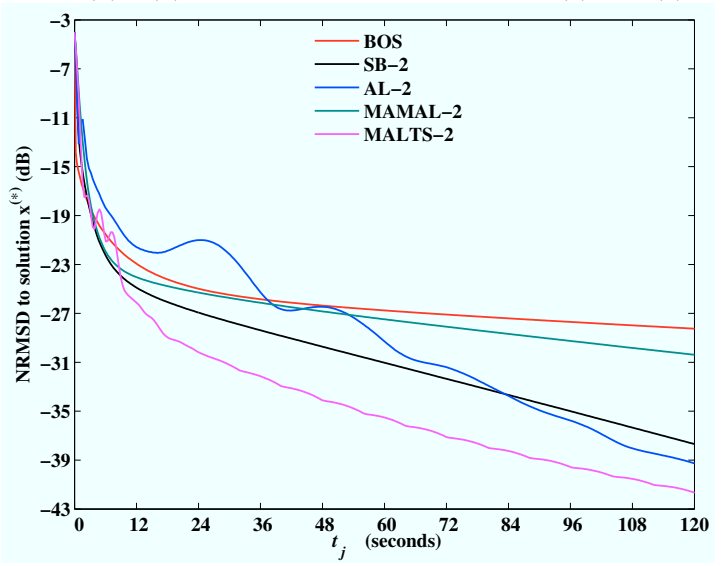

Fig. 4. Plot of NRMSD between $\mathbf{x}^{(j)}$ and a solution $\mathbf{x}^{(\star)}$ of P0 (1) for simulation with a BrainWeb [15] $T_{2}$-weighted MRI image.

to estimate $\mathbf{S}$ from $\mathbf{d}_{\text {cart }}$. We used an $\ell_{1}$-regularizer (2-levels of Haar frame for $\mathbf{R}$ ) and set $\gamma$ such that $90 \%$ of the elements of $\left(\mathbf{R} \mathbf{w}^{(1)}+\boldsymbol{\vartheta}^{(1)}\right)$ were nonzero after scalar-shrinkage operation at (12). The $256 \times 256 \ell_{1}$-regularized solution $\mathbf{x}^{(\star)}$ to $\mathbf{P 0}$ (1), Fig. $3 \mathrm{c}$, has reduced artifacts compared to $\mathrm{CPR}$, Fig. $3 \mathrm{~b}$, and is visually comparable to the noisefree Brainweb image, Fig. 3a, while the difference image, Fig. 3d, indicates that $\mathbf{x}^{(\star)}$ has relatively more (structured) residual error in this experiment. Fig. 4 plots NRMSD as a function of run-time for all the considered algorithms. BOS is the slowest, while the proposed MAMAL is slower than AL and SB. However, MALTS decreases NRMSD more rapidly than all other algorithms indicating its superior convergence speed.

\section{CONCLUSION}

We proposed an iterative algorithm for Regularized SENSE reconstruction for nonCartesian trajectories using the majorize-minimize (MM) principle and a variable splitting (VS) strategy [5]. Specifically, we designed a majorizer involving a circulant matrix for the SENSE data-fidelity term. Combined with the augmented Lagrangian (AL) framework and alternating minimization [5], this majorizer led to an iterative MAMAL algorithm that requires only one pair of NUFFT/iNUFFT per (outer) iteration, admits simple update steps that can be implemented efficiently or in closed-form and can efficiently tackle a variety of convex regularizers including $\mathrm{TV}$ and those based on the $\ell_{1}$-norm. We also incorporated a twostep (TS) strategy [6] to accelerate MAMAL: the resulting MALTS algorithm converged faster than some of the recent state-of-the-art VS-based algorithms such as the split-Bregman and the Bregman operator splitting methods. The proposed MALTS algorithm has potential for Regularized SENSE reconstruction for 3-D nonCartesian trajectories.

\section{REFERENCES}

[1] N. Seiberlich, F. A. Breuer, M. Blaimer, K. Barkauskas, P. M. Jakob, and M. A. Griswold, "Non-Cartesian data reconstruction using GRAPPA operator gridding (GROG)," Mag. Res. Med., vol. 58, no. 6, pp. 1257-65, Dec. 2007.

[2] J. A. Fessler and B. P. Sutton, "Nonuniform fast Fourier transforms using min-max interpolation," IEEE Trans. Sig. Proc., vol. 51, no. 2, pp. 560-74, Feb. 2003.

[3] K. P. Pruessmann, M. Weiger, P. Börnert, and P. Boesiger, "Advances in sensitivity encoding with arbitrary k-space trajectories," Mag. Res. Med., vol. 46, no. 4, pp. 638-51, Oct. 2001.

[4] L. Ying, B. Liu, M. Steckner, G. Wu, M. Wu, and S-J. Li, "A statistical approach to SENSE regularization with arbitrary kspace trajectories," Mag. Res. Med., vol. 60, no. 2, pp. 414-21, Aug. 2008.

[5] S. Ramani and J. A. Fessler, "Parallel MR image reconstruction using augmented Lagrangian methods," IEEE Trans. Med. Imag., vol. 30, no. 3, pp. 694-706, Mar. 2011.

[6] A. Beck and M. Teboulle, "A fast iterative shrinkagethresholding algorithm for linear inverse problems," SIAM J. Imaging Sci., vol. 2, no. 1, pp. 183-202, 2009.

[7] T. Goldstein and S. Osher, "The split Bregman method for L1regularized problems," SIAM J. Imaging Sci., vol. 2, no. 2, pp. 323-43, 2009.

[8] X. Zhang, M. Burger, X. Bresson, and S. Osher, "Bregmanized nonlocal regularization for deconvolution and sparse reconstruction," SIAM J. Imaging Sci., vol. 3, no. 3, pp. 253-76, 2010.

[9] A. Van Den Bos, "Complex gradient and hessian," IEE Proc. Vis. Im. Sig. Proc., vol. 141, no. 6, pp. 380-3, 1994.

[10] M. Guerquin-Kern, L. Lejeune, K. P. Pruessmann, and M. Unser, "Realistic analytical phantoms for parallel magnetic resonance imaging," IEEE Trans. Med. Imag., vol. 31, no. 3, pp. 626-36, Mar. 2012.

[11] T. F. Chan, "An optimal circulant preconditioner for Toeplitz systems," SIAM J. Sci. Stat. Comp., vol. 9, no. 4, pp. 766-71, July 1988.

[12] R. H. Chan and M. K. Ng, "Conjugate gradient methods for Toeplitz systems," SIAM Review, vol. 38, no. 3, pp. 427-82, Sept. 1996.

[13] M. I. Grivich and D. P. Jackson, "The magnetic field of currentcarrying polygons: An application of vector field rotations," Amer. J. Phys., vol. 68, no. 5, pp. 469-74, May 2000.

[14] M. J. Allison, S. Ramani, and J. A. Fessler, "Accelerated regularized estimation of MR coil sensitivities using augmented Lagrangian methods," IEEE Trans. Med. Img., DOI:10.1109/TMI.2012.2229711, in press.

[15] R. K-S. Kwan, A. C. Evans, and G. B. Pike, "MRI simulationbased evaluation of image-processing and classification methods," IEEE Trans. Med. Imag., vol. 18, no. 11, pp. 1085-97, Nov. 1999. 